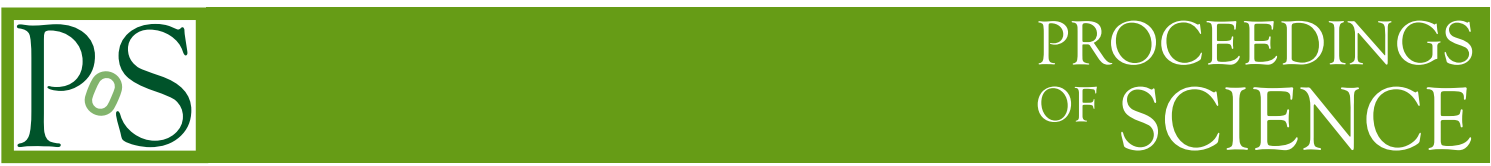

\title{
Status of the ATLAS experiment
}

\author{
Domizia Orestano* on behalf of the ATLAS collaboration \\ Dipartimento di Fisica "E.Amaldi" Università Roma Tre and INFN, Italy \\ E-mail: orestano@is.uniroma3.it
}

The ATLAS experiment at the CERN Large Hadron Collider was operated with colliding proton beams at $7 \mathrm{TeV}$ centre of mass energy since March 2010. It will study a broad range of particle physics phenomena at the highest available laboratory energies. At the time of the conference, ATLAS, with high data taking efficiency, had integrated a luminosity of few inverse picobarns, which allowed already observation and measurement of Standard Model processes, like vector boson and top production. Detector status, event reconstruction and particle identification performance in this first period of operation are presented together with selected first physics results.

Third International Workshop on Prospects for Charged Higgs Discovery at Colliders - CHARGED2010, September 27-30, 2010

Uppsala Sweden

${ }^{*}$ Speaker. 


\begin{tabular}{|c|c|c|c|c|c|c|c|c|c|c|}
\hline \multicolumn{3}{|c|}{$\begin{array}{l}\text { Inner Tracking } \\
\text { Detectors }\end{array}$} & \multicolumn{4}{|c|}{ Calorimeters } & \multicolumn{4}{|c|}{ Muon Detectors } \\
\hline Pixel & SCT & TRT & $\begin{array}{l}\text { LAr } \\
\text { EM }\end{array}$ & $\begin{array}{l}\text { LAr } \\
\text { HAD }\end{array}$ & $\begin{array}{l}\text { LAr } \\
\text { FWD }\end{array}$ & Tile & MDT & RPC & TGC & $\csc$ \\
\hline 97.7 & 96.4 & 100 & 94.4 & 98.7 & 99.3 & 99.2 & 98.5 & 98.3 & 98.6 & 98.3 \\
\hline
\end{tabular}

Table 1: Luminosity weighted relative fraction of good quality data delivery by the various ATLAS subsystems during LHC fills with stable beams in pp collisions at $\sqrt{s}=7 \mathrm{TeV}$, and after switching the tracking detectors on.

The ATLAS experiment [1] at the Large Hadron Collider (LHC) has been designed to study a wide spectrum of physics processes in proton-proton (pp) and heavy ions collisions at the highest centre of mass energies ever reached. This document summarises the status of the detector during data taking, the reconstruction and identification of physics objects [2] at the time of the Charged Higgs 2010 workshop, and provides some highlights of the first physics results based on pp collisions at $\sqrt{s}=7 \mathrm{TeV}$. This contribution reflects the state-of-the-art at a time in which the LHC luminosity was increasing rapidly, therefore the integrated luminosity collected up to that date was fully analysed only in few cases. Most of the results presented here are still based on a small subset of the available statistics.

\section{Data Collection}

LHC operation with protons at $\sqrt{s}=7 \mathrm{TeV}$ started in March 2010 and the beam intensity was gradually increased, first by raising the single bunch intensity and then by increasing the number of colliding bunches. At the time of the conference, in late September 2010, the instantaneous luminosity had reached few units in $10^{31} \mathrm{~cm}^{-2} \mathrm{~s}^{-1}$ and was rapidly raising towards $10^{32} \mathrm{~cm}^{-2} \mathrm{~s}^{-1}$, which was then reached on October 14th. Over all this period the ATLAS detector was operated smoothly and efficiently. Figure 1-left displays the integrated luminosity delivered by LHC and recorded by ATLAS up to September 25th, representing about one tenth of the value achieved at the end of the 2010 proton run.

The delivered luminosity accounts for the luminosity delivered from the start of stable beams until the LHC requests ATLAS to turn the sensitive detector off to allow a safe beam dump or beam studies. The luminosity reported here is determined from counting rates measured by the luminosity detectors. These detectors have been calibrated with the use of the Van-der-Meer beamseparation method, where the two beams are displaced one with respect to the other in the horizontal and vertical planes to measure their overlap function [3]. The systematic uncertainty of the absolute luminosity measurement is estimated to be $11 \%$, dominated by the uncertainty in the beam current product of $10 \%$. It should be mentioned that with the current machine settings already $40 \%$ of the bunch crossings produce more that one collision and this "pile up" cannot be neglected in the analyses.

Besides the high data collection efficiency, the whole ATLAS detector, readout through about 90 million channels, performed reliably, as certified by careful online and offline checks and summarised in table 1. 

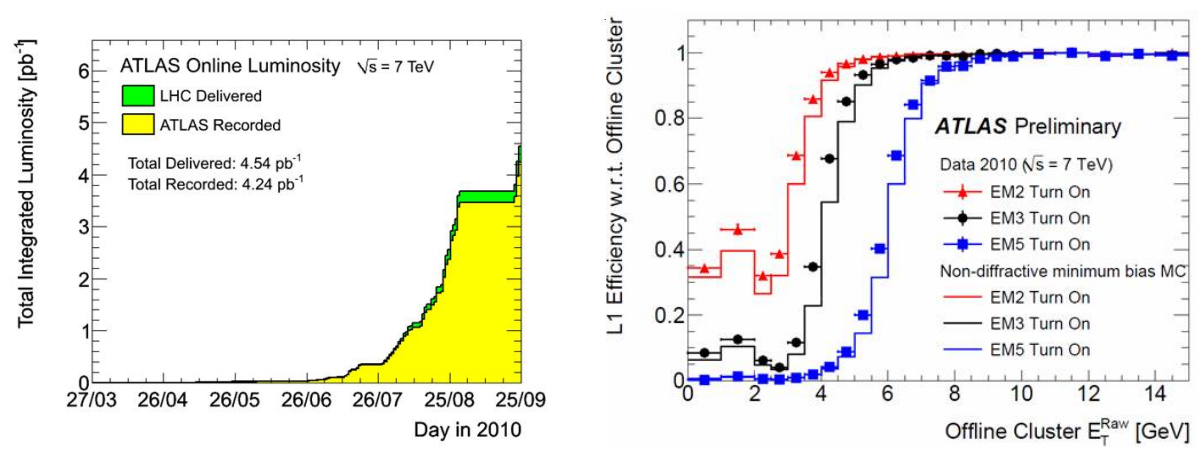

Figure 1: Cumulative luminosity versus day delivered to, and recorded by ATLAS during stable beams and for pp collisions at $7 \mathrm{TeV}$ centre-of-mass energy (left). An example of trigger efficiency determination: L1 electromagnetic trigger efficiency, for different thresholds, referred to offline reconstructed clusters (right).

\section{Trigger performance}

One of the main activities in the first data taking period has been the commissioning of the ATLAS three level trigger system. The first level (L1) uses custom electronics for a rapid selection of interesting events, while the next two levels (L2 and EF), known as high level triggers (HLT), apply a refined selection running software algorithms of increasing complexity on farms of commercial computers. Different "slices" involving all three levels are defined accordingly to the physics objects of interest and the applied thresholds. In order to progressively commission the trigger while coping with the rapidly increasing luminosity and the limited data taking bandwidth, a complex but flexible "trigger menu" was set up. This allowed to refer the performance of higher threshold triggers to lower level ones, or of HLT with respect to L1, or of a generic trigger with respect to an independent one, before raising the threshold or activating the selection through HLT. Figure 1-right shows one of the possible approaches to the trigger efficiency evaluation: the use of offline identified objects as reference in the case of a calorimetric trigger.

\section{Physics objects}

First data have also been used to tune reconstruction and understand identification of the basic physics objects, from charged particles to jets, electrons and photons, muons and taus, and of the derived ones, missing transverse energy and b-tagging techniques. The commissioning of tau identification, discussed in [4], will not be treated here.

\subsection{Charged particles}

The reconstruction of charged tracks in the inner detector, housed in a $2 \mathrm{~T}$ superconducting solenoid, proceeds in the central region (barrel) from the hits of three coaxial cylindrical detector systems. Moving away from the interaction point tracks cross the Pixel Detector, the Semiconductor Tracker (SCT) and the Transition Radiation Tracker (TRT). The same technologies are adopted in the two endcaps, where detectors are organised in discs. The coverage extends up to 2.5 in pseudo-rapidity $(\eta)$. 

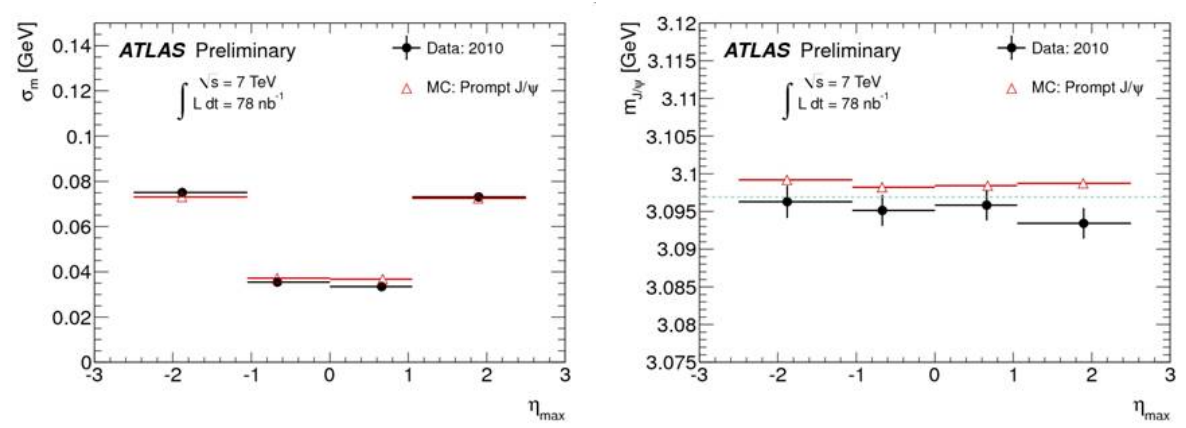

Figure 2: Reconstructed mass resolution of the $J / \Psi$ as a function of the pseudo-rapidity of the furthest forward muon (left) and reconstructed mass of the $\mathrm{J} / \Psi$ as a function of the pseudo-rapidity of the furthest forward muon (right). The dashed line corresponds the Particle Data Group value of the J/ $\Psi$ mass.
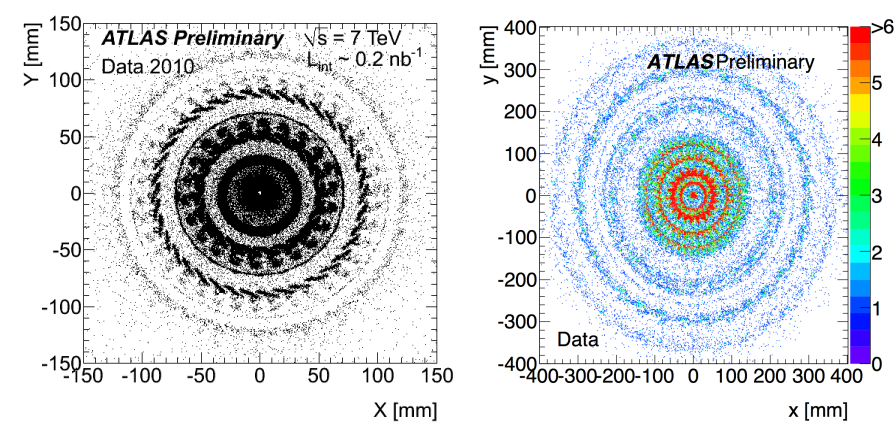

Figure 3: Zoomed-in view of the transverse position of reconstructed vertices after $K_{s}^{0}, \gamma$ conversion, and $\Lambda$ vetoes, and a $|Z|<300 \mathrm{~mm}$ cut on the longitudinal vertex position (left). The bin width is $1 \mathrm{~mm}$ in $\mathrm{X}$ and $\mathrm{Y}$. Distribution of the reconstructed conversions for data events as a function of the transverse positions of the reconstructed conversion vertices (right).

Track reconstruction efficiency has been extensively studied in pp collisions in the context of the measurement of charged particles production at the different centre of mass energies explored at $\mathrm{LHC}(0.9 \mathrm{TeV}$ [5], $2.36 \mathrm{TeV}, 7 \mathrm{TeV})$ and is well modelled by the detector simulation.

Momentum bias and resolution, very sensitive to the internal alignments of this complex system, have been monitored using resonances, and in particular with $K_{s}^{0}$ and $\mathrm{J} / \Psi$ decays. Figure 2 illustrates the performance measured on a sample of about $5000 \mathrm{~J} / \Psi$ decays into muons, compared to Monte Carlo expectations. While the mass resolution is in good agreement between data and Monte Carlo simulation, the reconstructed mass exceeds systematically the Particle Data Group value in simulation and is systematically lower in data.

The resolution and mass of the reconstructed $K_{s}^{0}$ decays allowed also, in comparison to the simulation, to check the description of the amount of material crossed by the two pion tracks. A data driven estimate of the amount and distribution of the material can also be obtained through the distribution of reconstructed secondary interactions or photon conversion points, as shown in figures 3. In fact tracking provides also vertex reconstruction, both for primary and secondary vertices, together with the possibility to measure track's impact parameter with respect to the primary vertex. 

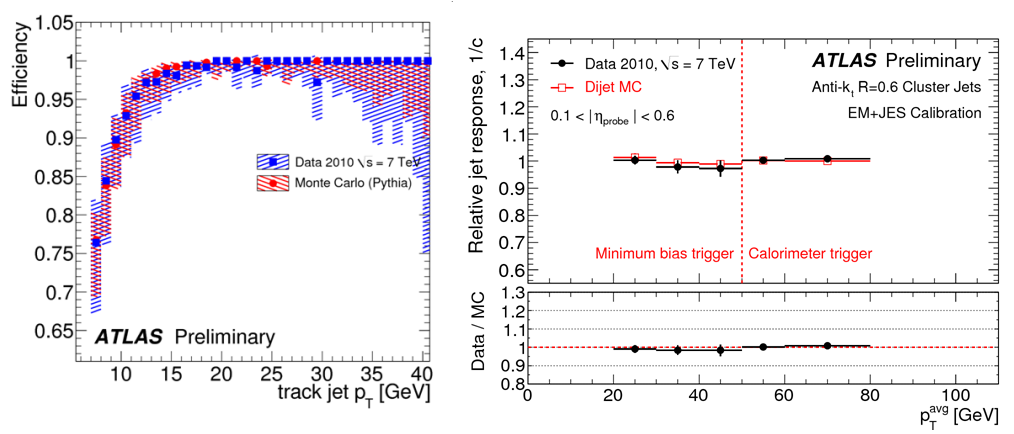

Figure 4: Efficiency of calorimetric jets referred to tracking ones (left) and relative jet response in dijet events (right).

\subsection{Jets}

Jet reconstruction can be based either on calorimetric information or on tracking. The baseline jet definition in ATLAS relies on calorimetric information and reconstruction through the AntiKt algorithm [6]. The track jets are used for instance to monitor the efficiency of the calorimetric ones, as shown in figure 4-left. The ATLAS calorimeter does not compensate for the different energy released by the hadrons and the electromagnetic component. Corrections for this effect are obtained in fine $\eta$ and transverse energy bins from Monte Carlo. The performance in data is evaluated using data driven techniques. For instance figure 4-right shows the relative jet response obtained after calibration in dijet events, where the reference jet is selected at low pseudo-rapidity, in a region where the calorimetric response is more uniform. Figure 5 shows some inclusive jet and dijet distributions, illustrating the good agreement with Monte Carlo expectations over more than five decades, up to transverse energies and invariant masses above the TeV. Quantum Chromodynamics (QCD) cross-sections measured in ATLAS and compared to Next to Leading Order (NLO) calculations and various Monte Carlo predictions are published in [7].
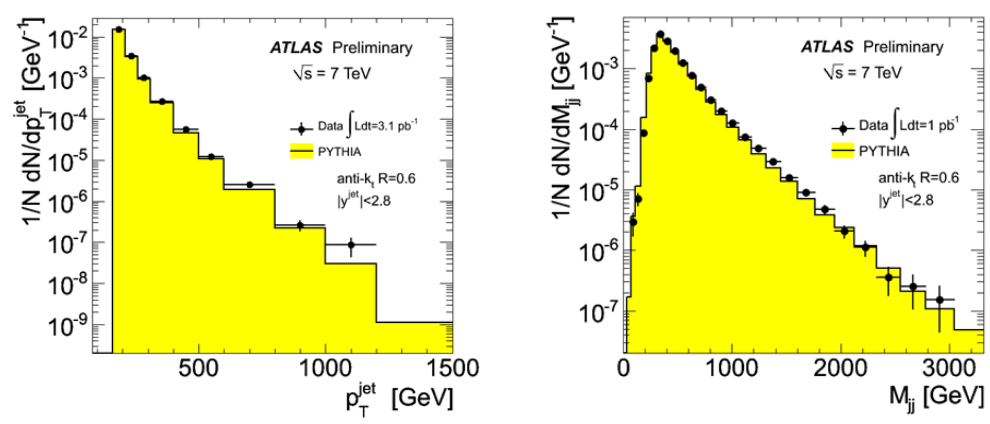

Figure 5: Observed inclusive jet transverse momentum distribution (dots) for jets with $P_{T}($ jet $)>160 \mathrm{GeV}$ and $\mid \eta$ (jet) $\mid<2.8$ (left) and dijet invariant mass distribution (dots) for jets with leading $P_{T}($ jet $)>160 \mathrm{GeV}$ and second-leading $P_{T}($ jet $)>40 \mathrm{GeV}$, with $\mid \eta($ jet $) \mid<2.8$ (right). Distributions are compared to PYTHIA Monte Carlo prediction (histogram), are normalized to unity and only statistical uncertainties are included.

\subsection{Electrons and photons}

Electrons and photons represent important signatures of interesting physics but their identifi- 

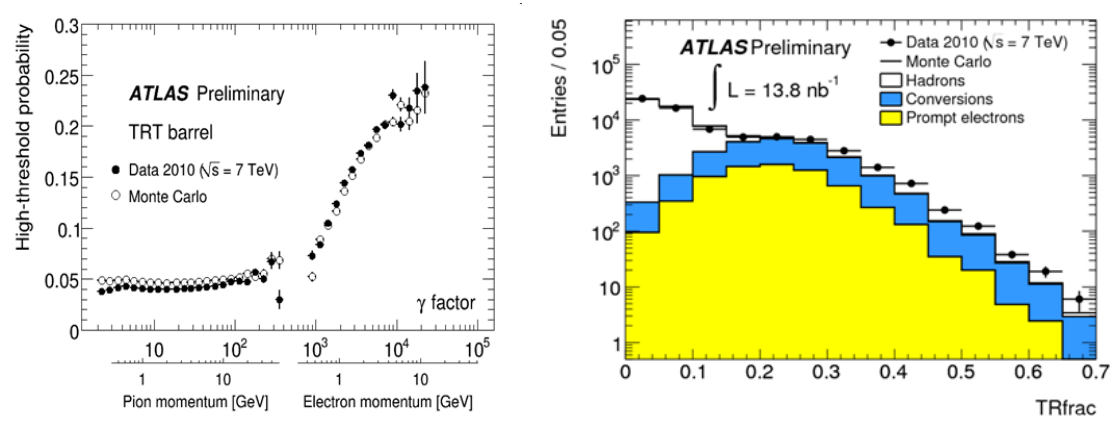

Figure 6: TRT response to pions and electrons measured in data and compared to Monte Carlo (left). Distribution of the fraction of TRT hits above the high threshold in an inclusive electron sample compared to the different sources of electron candidates (right).

cation is particularly challenging due the high background from hadrons, affecting both electrons and photons, and from conversions, affecting the electrons. The reconstruction of electromagnetic objects is based on the electromagnetic calorimeter, built with an accordion geometry adopting liquid Ar as sensitive medium and lead as absorber. Starting from seed clusters with significant energy above the noise, electromagnetic clusters are formed and the matching with charged tracks is attempted. Depending on the outcome, candidates are classified as electrons or unconverted photons. Converted photons are identified on the basis of the absence of hits in the innermost layer of the tracker. The transverse and longitudinal profiles of the shower, thanks to the fine segmentation of the calorimeter, provide discriminant variables which can be used to improve the purity after the first step of identification. In the case of the electrons the discrimination against hadrons is further enhanced by the detection of transition radiation, produced in polypropylene foils in the TRT. The probability of hits above the high threshold to be produced by electrons and pions is shown in figure 6 together with the distribution of the fraction of TRT hits above that threshold for an inclusive electron sample.

\subsection{Muons}

Muons are identified through their capability of penetrating through the calorimeters and reach the muon spectrometer. The deflection of muons by the magnetic field generated by a system of air core toroid coils in the muon spectrometer is measured by three layers of precision gaseous detector up to $|\eta|<2.7$. The position in the non bending plane is provided by the muon trigger detectors. Up to $|\eta|=2.5$ muon momenta are also measured in the inner detector. Muon performance evaluation in first data has been focused on efficiency, fake rate and resolution measurements, and was limited by the available statistics to transverse momenta up to $\approx 15 \mathrm{GeV}$. Relative efficiencies have been studied on single muons exploiting the redundancy of detector technologies and reconstruction algorithms and the minimum ioninizing particles identification in the calorimeters. Fake rates studies are based on the mis-tag rate for pions and kaons identified in hadronic resonances $\left(K_{S}^{0}\right.$ and $\left.D^{*}\right)$ and on the momentum unbalance between inner detector and muon spectrometer measured momenta, due to late decays of pions and kaons. Relative resolution, between inner detector and muon spectrometer, has been studied on single muons and was found in agreement for the barrel region with the measurements obtained in cosmic ray runs. The transverse momentum range covered 

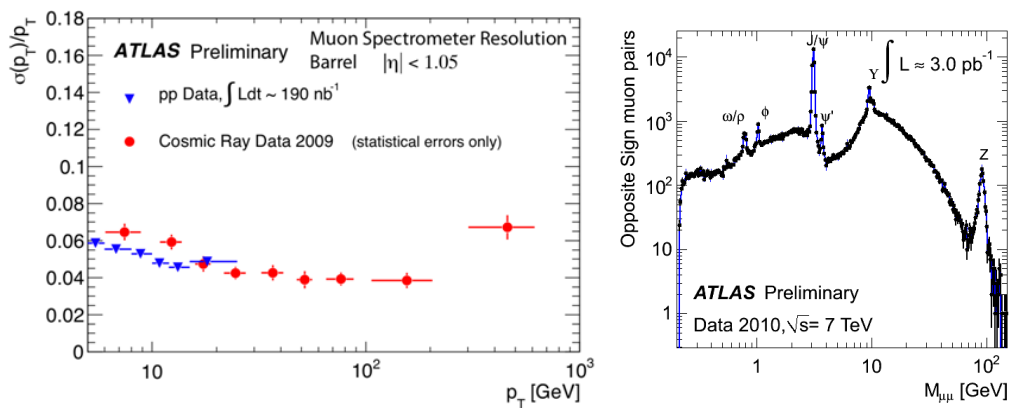

Figure 7: The muon spectrometer resolution relative to the inner detector (whose contribution is neglected) in the barrel, compared to Cosmic Ray results (left). The opposite sign dimuon invariant mass for an integrated luminosity of $3 \mathrm{pb}^{-1}$ collected with the first-level muon trigger .
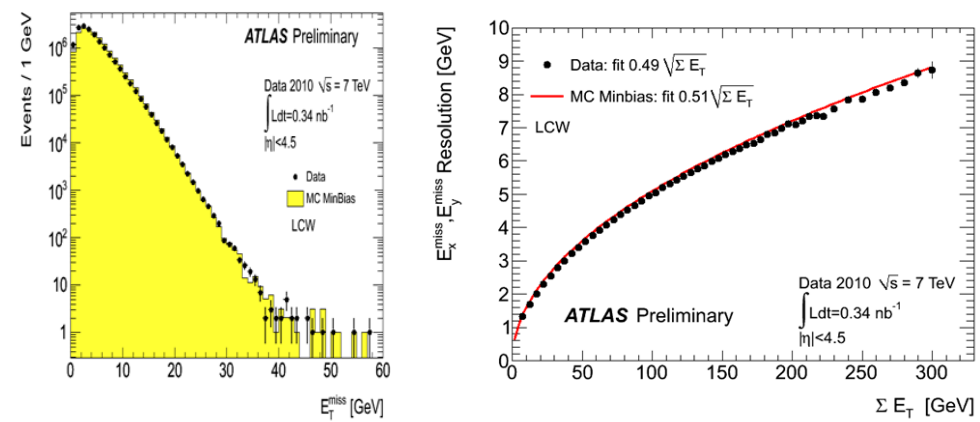

Figure 8: Distribution of the missing transverse energy reconstructed in minimum bias events compared to Monte Carlo expectations (left). Missing transverse energy resolution in data and Monte Carlo as a function of the total transverse energy in the event (right).

by these studies, shown in figure 7-left, is limited by the statistics and has been further extended in more recent results.

With the statistics analysed after this conference absolute resolutions and efficiencies were obtained exploiting the clean signatures of $\mathrm{J} / \Psi$ and $\mathrm{Z}$ decays to dimuons (see figure 7-right). Results of these more detailed studies were found in agreement with the above ones.

\subsection{Missing transverse energy}

Missing transverse energy in an event is a measure of the energy imbalance in the transverse plane and it indicates the presence of undetected particles (like neutrinos). Such imbalance may also rise from detector effects or relative miscalibration of the scales of the many detectors. Being measured from all particles seen in the detector, it is expected to scale with the amount of energy in the event. The key performance figures are the transverse energy resolution, shown in figure 8-right as a function of the total transverse energy, and the tails of the missing transverse energy distribution in minimum bias events, not expected to show any significative enhancement with respect to Monte Carlo, as confirmed by figure 8 -left. 

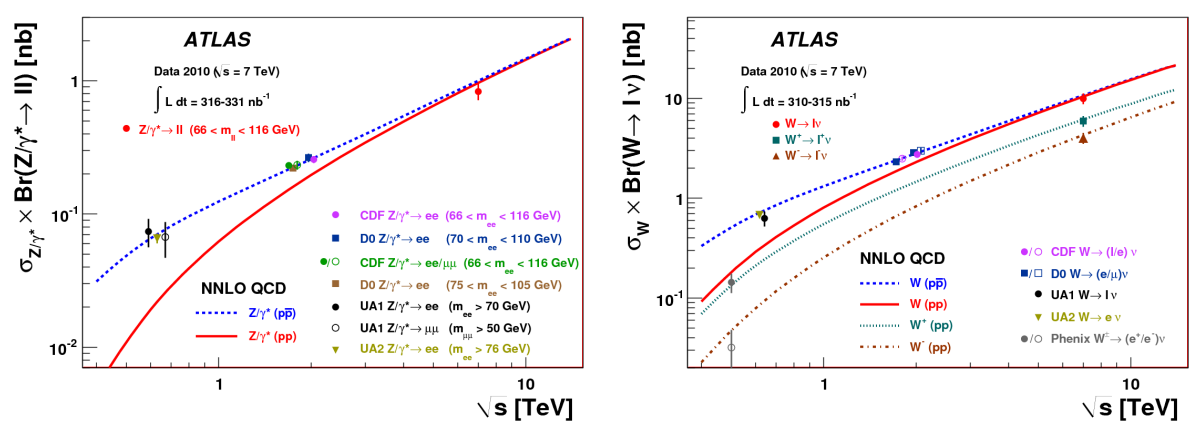

Figure 9: The measured value of $\mathrm{W}$ (left) and $\mathrm{Z} / \gamma^{*}$ (right) cross sections times branching ratios in electron and muon channels combined. Predictions based on NNLO QCD calculations are shown for both protonproton and proton-antiproton colliders. The data points from previous experiments, at the various energies, are staggered to improve readability.

\subsection{Tagging of $b$ jets}

The identification of jets originating from b-quarks is an important part of the LHC physics program. It exploits the long lifetime of $b$ flavoured particles and their high mass, through secondary vertices, high impact parameter tracks, transverse momentum of $\mathrm{b}$ decay products with respect to the jet axis. In ATLAS two types of algorithms have been commissioned: the SV0 algorithm, which is a lifetime-based tagging algorithm relying on the reconstruction of secondary vertices within jets, and the algorithms using displaced tracks near jets which likely originate from b-quarks. The possibility of tagging b-jets through the presence of a muon with transverse momentum with respect to the jet axis higher than the typical values detected in light jets, is used in the performance evaluation of the previously explained techniques.

\section{First results}

With the integrated luminosity analysed at the time of the conference ATLAS is not yet competitive with Tevatron experiments but for searches of heavy mass states. For this reason among the first physics results, besides the cross-section measurements for $\mathrm{W}$ and $\mathrm{Z}$ production, only the search for new particles in two jet final states will be presented.

\section{1 $\mathrm{W}$ and $\mathrm{Z}$ production}

Measurements of the $W \rightarrow l v$ and $Z \rightarrow l l(1=\mathrm{e}, \mu)$ production cross sections in pp collisions at $\sqrt{s}=7 \mathrm{TeV}$ have been obtained from the first $\approx 320 \mathrm{nb}^{-1}$ of integrated luminosity [8]. Results are based on $2250 \mathrm{~W} \rightarrow l \mathrm{~V}$ and $179 \mathrm{Z} \rightarrow l l$ candidate events.

The measured total $\mathrm{W}$ and $\mathrm{Z}$ production cross sections times the respective leptonic branching ratios for the combined electron and muon channels are presented in figures 9 . The cross sections for $W^{+}$and $W^{-}$have been measured separately together with the asymmetry in lepton charge, $A=\frac{\sigma\left(l^{+} v\right)-\sigma\left(l^{+} v\right)}{\sigma\left(l^{+} v\right)+\sigma\left(l^{+} v\right)}$, see figure 10. Theoretical predictions based on Next to Next Leading Order (NNLO) QCD calculations are found to agree with all the above measurements. 

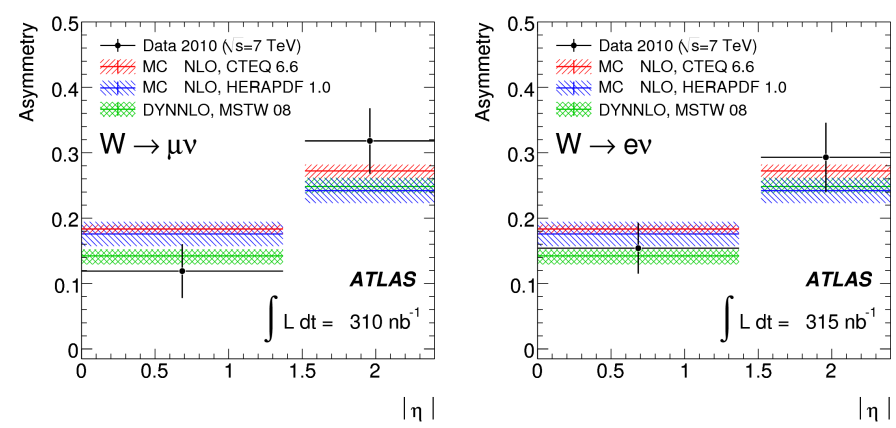

Figure 10: Lepton charge asymmetries for the electron (left) and muon (right) channels. Superimposed are several theoretical predictions with bands obtained from different sets of PDFs.

\subsection{Dijet invariant mass spectrum}

Dijet events in pp are described in the Standard Model (SM) by applying QCD to the scattering of beam-constituent quarks and gluons. Several extensions beyond the SM predict new heavy particles, accessible at LHC energies, that decay into two energetic partons. These states should manifest themselves through a distortion of the dijet invariant mass spectrum with respect to QCD expectations. The analysis presented here focuses on the search for excited quarks, whose predicted cross sections were accessible already with the few hundred inverse nanobars of integrated luminosity for which first results have been published [9]. The analysis is based on a sample of jet triggered events with $15 \mathrm{GeV}$ transverse energy threshold reconstructed using the AntiKt jet algorithm with radius 0.6. Events with at least two jets of transverse momentum above $80 \mathrm{GeV}$ and $30 \mathrm{GeV}$, satisfying some quality criteria, and having invariant mass above $200 \mathrm{GeV}$, were retained. Figure 11-left shows the result of the fit with a smooth background distribution validated on Monte Carlo and the expected signal contributions of excited-quark of different masses from Monte Carlo. The good agreement between data and the background-only hypothesis allowed to set the 95\% CL limit on the cross section times acceptance shown in figure 11-right as a function of the resonance mass. From the comparison to Monte Carlo predictions ATLAS excludes at 95\% CL excited-quark states decaying into two jets with masses below $1.53 \mathrm{TeV}$.

\section{Conclusions}

The first six months of LHC operation at $\sqrt{s}=7 \mathrm{TeV}$ have provided ATLAS and the other LHC experiments with enough luminosity for a variety of performance studies and few first physics measurements. Some results are addressing the "rediscovery" of Standard Model signatures and the tuning of the Monte Carlo models, while few are already extending beyond the domain explored by Tevatron in the search for new physics. The turn on point in 2010 LHC run was right at the time of this conference, when in a few weeks period the integrated luminosity grew by a factor 10 . This contribution reflects the state-of-the-art just before this large increase in statistics, but testifies already of the brilliant operation both of LHC and ATLAS. 

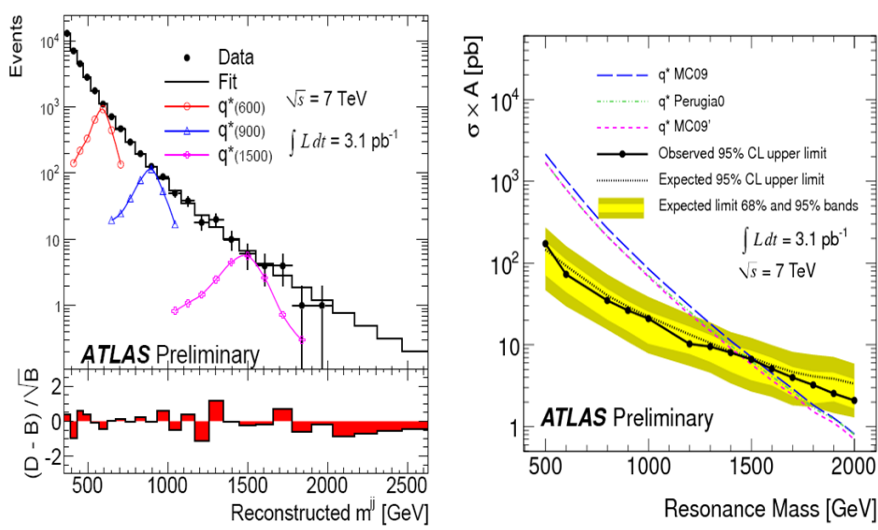

Figure 11: The data dijet mass distribution fitted using a smooth binned background distribution. The predicted signals for excited-quark masses of 600,900 , and $1500 \mathrm{GeV}$ are over- laid, and the bin-by-bin significance of the data-background difference is shown (left). The 95\% CL upper limit on $\sigma \times A$ as a function of dijet resonance mass. The dotted curve shows the expected 95\% CL upper limit and the light and dark shaded bands represent the $68 \%$ and $95 \%$ credibility intervals of the expected limit, respectively. The dashed curves represent predictions for excited-quarks for different MC tunes, each using a different PDF set (right).

\section{References}

[1] ATLAS Collaboration, G. Aad et al., The ATLAS Experiment at the CERN Large Hadron Collider, JINST 3 (2008) S08003.

[2] Documentation on the performance studies addressed in this contribution can be found in https://twiki.cern.ch/twiki/bin/view/Atlas/AtlasResults and in http://cdsweb.cern.ch/collection/ATLAS20Conference20Notes.

[3] ATLAS-CONF-2010-060 (2010) [http://cdsweb.cern.ch/record/1281333].

[4] Y.Coadou, Tau trigger and tau reconstruction, efficiency and fake rates in ATLAS, these proceedings.

[5] G. Aad et al. (ATLAS Collaboration), Charged-particle multiplicities in pp interactions at sqrt $(s)=$ $900 \mathrm{GeV}$ measured with the ATLAS detector at the LHC, Phys. Lett. B688, 21-42 (2010). [arXiv:1003.3124 [hep-ex]].

[6] M.Cacciari and G.Salam, The AntiKt jet clustering algorithm, 2008 [arXiv:0802.1189v2 [hep-ph]].

[7] ATLAS Collaboration, Measurement of inclusive jet and dijet cross sections in proton-proton collisions at $7 \mathrm{TeV}$ centre-of-mass energy with the ATLAS detector, in press [arXiv:1009.5908 [hep-ex]]

[8] ATLAS Collaboration, Measurement of the $W \rightarrow l v$ and $Z / \gamma^{*} \rightarrow l l$ production cross sections in proton-proton collisions at $\sqrt{s}=7 \mathrm{TeV}$ with the ATLAS detector, in press [arXiv:1010.2130 [hep-ex]].

[9] ATLAS Collaboration, Search for New Particles in Two-Jet Final States in 7 TeV Proton-Proton Collisions with the ATLAS Detector at the LHC, Phys. Rev. Lett. 105, 161801 (2010), [arXiv:1008.2461 [hep-ex]]. 